\title{
Design and deployment of relational geodatabase on mobile GIS platform for real-time COVID-19 contact tracing in Ghana
}

Seth Kwaku Afagbedzi ${ }^{1}$, Alex Barimah Owusu2*, Isaac Newton Kissiedu², Mary Amoako- Coleman ${ }^{3}$,

Delia Akosua Bandoh ${ }^{4}$, Charles Lwanga Noora ${ }^{4}$, Ben Emunah Aikins ${ }^{2}$, Richmond Takyi Hinneh²,

Benedict Calys-Tagoe ${ }^{5}$, Keziah Laurencia Malm ${ }^{6}$, Ernest Kenu ${ }^{4}$

\begin{abstract}
This study reviewed the design and deployment of relational geodatabase on mobile GIS application, using collector for ArcGIS and survey 123 for ArcGIS platforms for COVID-19 contact tracing in Ghana during the lockdown. The study assessed whether cases spread by physical neighborhood contacts, defined by a $2 \mathrm{~km}$ buffer of initial known 60 cases location. The application was deployed on the android tablet, which was used by field workers. Application Post-deployment review shows that from $30^{\text {th }}$ March to $4^{\text {th }}$ April 2020, 828 samples were collected with 34 confirmed cases, of which $61 \%$ occurred outside the $2 \mathrm{~km}$ buffer. From 1-30 April 2020, 8,748 individuals with 16,087 contacts were tested within the physical neighbourhoods, $2.4 \%$ turned positive. Similarly, 7,501 individuals with 17,071 contacts were tested outside the physical neighbourhoods with $4.3 \%$ positives. Results suggest that more infections occurred outside the case's physical neighbourhoods possibly due to; (1) existence of unknown cases prior to lockdown; (2) cases were moving outside their physical neighborhood and infecting others; (3) panic movements of cases within the 3 days window between announcement and enforcement of lockdown; (4) movement of cases into the country through unapproved routes. New cases were identified outside the lockdown areas, which could not be explained. This study raises questions about (1) the understanding of the mode of spread of the virus (2) the implementation of the lockdown, including the geographic coverage and timing. It is recommended that future decisions on contact tracing and lockdown should be guided by an understanding of the disease geography.
\end{abstract}

Keywords: Mobile geospatial technology, Novel Coronavirus 2019, Contact Tracing, COVID-19 in Ghana, Relational Geodatabase, GIS

\footnotetext{
${ }^{1}$ University of Ghana, Department of Biostatistics ${ }^{2}$ University of Ghana, Department of Geography and Resource Development *Corresponding Author. Email: owusuba@yahoo.com ${ }^{3}$ Department of Epidemiology, NMIMR, University of Ghana ${ }^{4}$ Department of Epidemiology and Disease Control, School of Public Health, University of Ghana ${ }^{5}$ Department of Community Health, University of Ghana Medical School ${ }^{6}$ National Malaria Control Programme, Ghana Health Service, Accra, Ghana
}

Ghana Journal of Geography Vol. 13 (1), 2021 pages 126-146

https://dx.doi.org/10.4314/gjg.v13i1.7 


\section{Background}

The Novel Coronavirus 2019 (COVID-19) also known as severe acute respiratory syndrome coronavirus 2 (SARS-CoV-2) has become the biggest pandemic the world has witnessed since the 1918 Flu (Spanish Flu) pandemic which infected approximately a quarter of the world's population and left between 17 and 50 million dead (AS, 2020). The disease has devastated the whole world by killing over 500,000 people as at the end of June 2020 (WHO, 2020), disrupted supply chains, caused lockdown of countries and cites, grounded many flights the world over and brought the global economy to a halt (Ritchie e. al., 2020). All over the world, governments are implementing various fiscal measures to mitigate the adverse effect and provide relief for businesses and households (ILO, 2020). The pandemic is expected to have a significant adverse impact on the global economy, which is estimated to be over $\$ 3$ trillion (Chaturvedi, 2020). Countries are in dilemma to re-open their economies amid soaring new infections and deaths. Due to limited knowledge and understanding of COVID-19, the global approach was organized in two ways; (a) medium to long term efforts which were geared toward developing vaccines since COVID-19 has no known cure (Chatham House, 2020); (b) the short-term strategies, which were focused on prevention of the spread through early detection, diagnosis, prompt contact tracing and isolation to extinguish the spread of infections. The short-term effort requires answering questions relating to the location of infected persons; i.e. where is the virus? Where is it spreading to, and where is one likely to contract the virus? Identifying the exact location of COVID-19 patients and contacts, monitoring and tracking their movements, and isolating them, minimizes the need to impose lockdowns involving mobility restrictions or business closures (Torhonen et. al, 2020). The short-term effort required timely and accurate data, which is spatially referenced and temporally current.

The need for spatially referenced and temporally current data to manage and control the spread of the COVID-19 pandemic highlighted the necessity for the geospatial community to support the national response efforts to curb the imminent public health threat brought about by this disease (Malgorzata and Pomfret, 2020). In the past, the geospatial industry has assisted during times of crises and disasters by improving relief and reintegration efforts (Herold and Sawada, 2012; Nkansah, 2020). With the advent of the coronavirus, the geospatial community is in the frontline providing data for monitoring and tracking the spread of the virus, continually bringing up-to-date data on the number of people infected and providing real-time information (Torhonen et. al., 2020). The geospatial community in Korea made use of Big Data, including 
GPS tracking data from cars and phones, transactions and credit card, CCTV footage, travel histories, and artificial intelligence to identify high-priority cases and track the routes of infected individuals (Chung and Hoon, 2020). Center for Systems Science and Engineering (CSSE) at Johns Hopkins University (JHU) with support from ESRI and other partners developed COVID-19 dashboard which monitors the ongoing COVID-19 globally by reporting on key indicators such as; the number of confirmed cases, deaths, recoveries and active cases by countries and globally (GHS, 2020). China has adopted several technologies including the COVID-19 status rating and monitoring app that present a QR code on the user's mobile phones. The app requires users to register with basic information - name, national identity card number, phone number, and home address. Other information on the app quizzes users on health status and travel history, and asks them to identify any close contacts diagnosed with the virus. A green QR code grants unrestricted movement, a yellow code required seven days of quarantine and a red meant 14 days of quarantine (Xu and Chandler, 2020).

As the pandemic surges on, governments and health authorities all over the world are relying on measures that are characteristically spatial such as quarantining, contact tracing, isolating and social distancing. While people are working and studying at home, decision-makers were exploring ways to circumnavigate the burdens of needing to prevent more infection, while also looking to relax restrictions and reopen the economy. Geospatial technologies are needed to enhance these processes promptly. Ghana's geospatial industry has equally contributed to the development and deployment of location-based mobile geospatial applications in combating the COVID-19 pandemic.

Ghana's fight against the pandemic, including control and management, has been directed from the presidency due to the seriousness of the disease and the urgent attention that it needed. Figure 1 shows Ghana's COVID-19 Governance Structure. The all-embracing governance and coordination of the National Covid-19 response start from the President of the Republic of Ghana. The Minister for Health chairs the National Technical Coordinating Committee (NTCC) at the ministerial level, which comprises representatives from all stakeholder ministries and relevant agencies to ensure a multi-sectoral approach and response. The NTCC provides the Technical policy direction and implementation of decisions made by the InterMinisterial group. The Ministry of Health and Ghana Health Service collaborate with other COVID-19 related task teams including the Operation COVID-19 Safety Taskforce. A decentralized approach to implementation from the National to Regional, District and Subdistrict levels is adopted to combat the pandemic. 


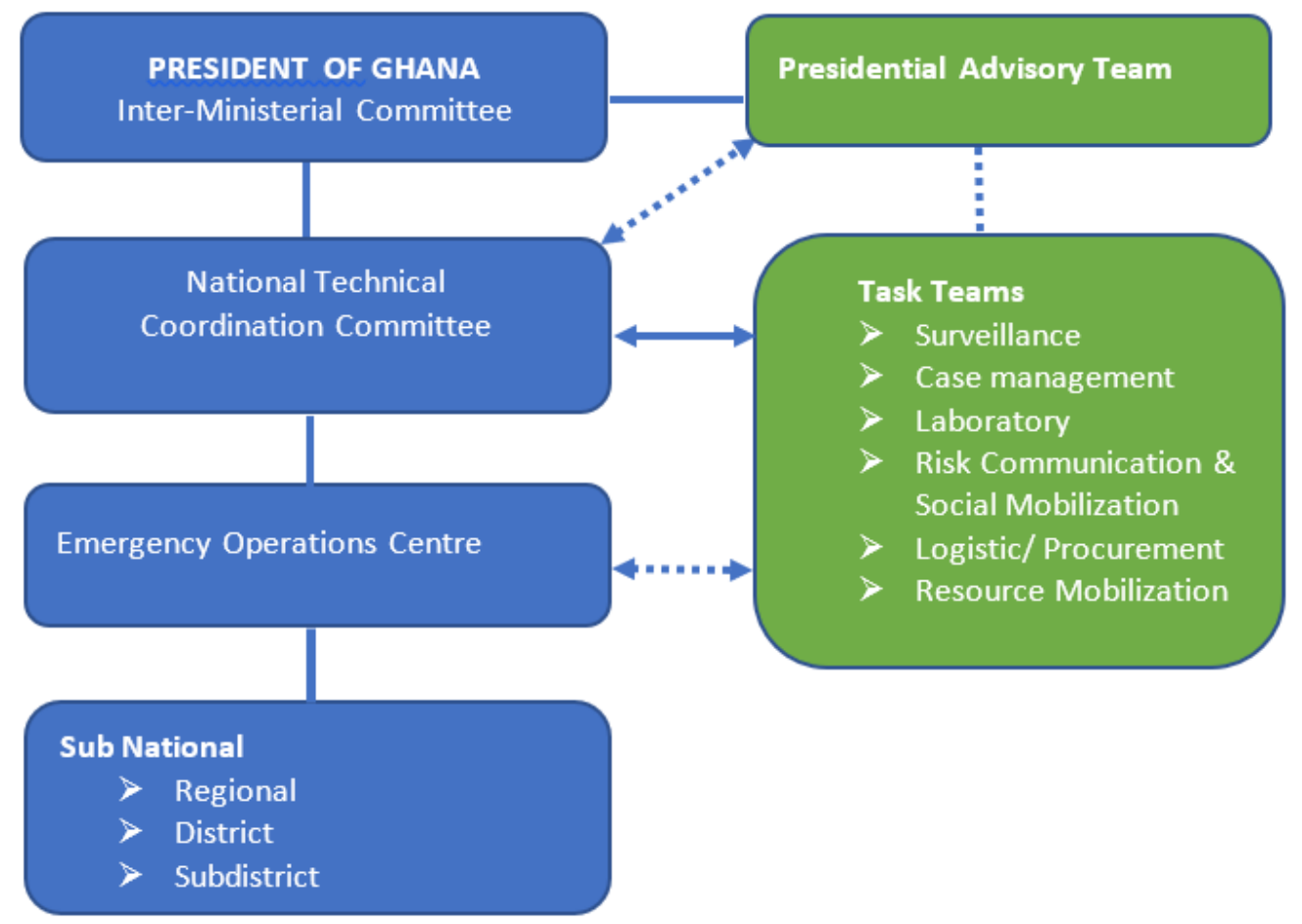

Figure 1: Ghana's COVID-19 Governance Structure

Source: National Strategic COVID-19 response plan, 2020-2021 - Ghana

The surveillance task team included monitors, contact tracers, epidemiologist, data managers and geospatial scientists. The geospatial team from the School of Public Health and the Remote Sensing and Geographic Information Systems Laboratory (RSGIS Lab) of the Department of Geography and Resource Development, - University of Ghana developed an integrated multiple geospatial application to support both the contact tracing and the enhanced contact tracing strategies adopted by Ghana during the early stages of the pandemic. With the enhanced contact tracing, a $2 \mathrm{~km}$ radius buffer zone (referred to as physical neighbourhood) was created around each of the initial COVID-19 cases and all household that fell within the buffered zone were screened for COVID-19 including the actual contacts to cases. This buffer was to make sure that every possible case that might be missed by the main contact-tracing list was captured to prevent the community spread of the virus. This mobile geospatial technology was one of the technologies used in the early stages in monitoring and tracking the spread of the disease in Ghana. Additionally, using collector for ArcGIS and survey 123 for ArcGIS platforms, a relational geodatabase was designed and deployed on mobile phones and tablets for contact tracing and enhanced contact tracing to provide spatially reference data in real-time to address 
the question of the whereabouts of the virus. The above two applications provided backend relational geodatabase and frontend customization that allowed data collection, storage, data query, and manipulation and visualization on dashboards to support field operations, laboratory testing of samples and official decision making on COVID-19 in Ghana (Guitierrez et al., 2007).

The primary aim of this paper is to review and demonstrate the relational geodatabase design and deployment for proactive spatial tracking of the spread of the virus and provision of realtime information for timeous decision making by the managers of the pandemic in Ghana. The propositions that guided the relational geodatabase design and deployment are:

Null Hypothesis 1: that Covid-19 will spread only by physical neighbourhood contacts during the lockdown period.

Alternate Hypothesis 2: that Covid-19 will not spread only by physical neighbourhood contacts during the lockdown period.

These hypotheses were made at the time of designing and deploying the integrated multiple geospatial application when there were 60 known cases of COVID-19 in Ghana, which was on the $27^{\text {th }}$ day of March 2020 (GHS, 2020). In addition, four (4) days after the government of Ghana announced COVID-19 restriction measures, which included the closure of the country's external borders and lockdown of the two main metropolitan areas: The Greater Accra Metropolitan Area (GAMA), the Greater Kumasi Metropolitan Area (GKMA), and the contiguous districts. With the lockdown, the country instituted limited mobility within the lockdown zone (Nkansah, 2020). Also, international borders of the country were closed and given that the cases in Ghana were all imported, the expectation was that cases will spread from the detected cases in their neighbourhoods (defined by $2 \mathrm{~km}$ Euclidean distance from case point location i.e. house or residence) and grow spatially outward (Nkansah, 2020). The $2 \mathrm{~km}$ distance was based on the standard routine activity range of an average Ghanaian in residential neighbourhoods. In addition, within the $2 \mathrm{~km}$ walking neighbourhoods, lockdown enforcing agencies were not questioning people. However, people who moved outside the walking neighbourhoods and start driving were questioned by COVID-19 restrictions enforcing agencies on their movement. The null hypothesis was tested against the alternative that the spread may not necessarily be physical neighbourhood contact. There may be other physical contacts engineered by socio-economic and political movements, even under the lockdown. Similarly, there may be other cases that were not detected, that could spread the virus. Also, it 
was likely that some importation may have occurred through illegal border crossing during the lockdown which might have not been detected.

\section{Methods}

\section{Study Setting}

This study is based on Ghana's initial approach to managing the COVID-19 pandemic. On $12^{\text {th }}$ March 2020, Ghana recorded the first two COVID-19 cases. The two index cases of COVID19 were confirmed in Accra on the same day and by $27^{\text {th }}$ of March 2020, there were 60 confirmed cases and 3 deaths (GHS, 2020). These cases were concentrated in GAMA and GKMA, which were referred to as COVID-19 hotspots. Figure 2 is the map of Ghana showing GKMA and GAMA inset. GAMA and GKMA cover several municipalities and metropolis including AMA and KMA respectively. On $27^{\text {th }}$ March 2020, the Government of Ghana (GoG) decided to partially lockdown the two metropolitan areas. AMA is the National capital and largest city in the Greater Accra Region, with a population of more than 2 million people (ILO, 2020). It houses the only international airport in Ghana, the Kotoka International Airport. As a result, AMA is the first and last point of call for all visitors and residents who travel in and out of Ghana. AMA is the administrative, economic, and educational capital of Ghana. The city contains the head offices of all the large banks and trading firms, the insurance agencies, the utility corporations, the general post office, the large open markets to which most of the food supply comes, and the Accra Central Library. AMA is a modern city with some of the best hotels, beaches and public places that attract many tourists making it most likely a COVID-19 transit city.

GKMA is the second metropolitan area identified with the initial COVID-19 cases and was therefore, affected by the lockdown. KMA (Kumasi) is the second-largest city in Ghana and is the most populous city in GKMA. The metropolis is the most populous in Ghana with an estimated population of over 2 million residents. The city of Kumasi is the hub of Ghanaian cultural heritage, which attract thousands of both domestic and foreign tourists yearly. The city has the most vibrant domestic travel activities by both air and road, hence its position as a high COVID-19 risk city. 


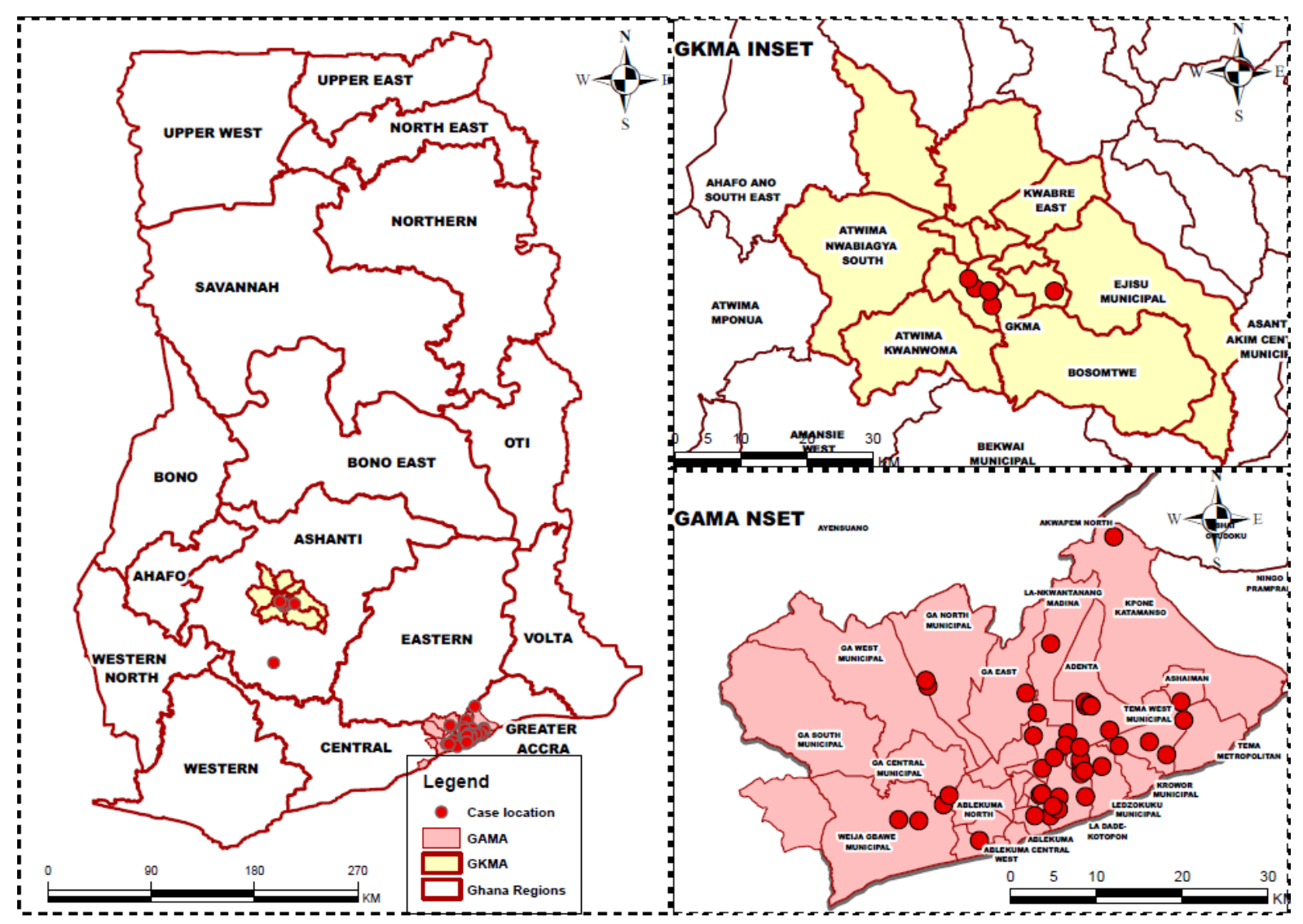

Figure 2: Map of Ghana with GAMA and GKMA Insets and location of the initial 60 COVID 19 Cases Source: Author 2020

\section{Design and deployment of the relational geodatabase on the mobile GIS system}

The design and deployment of the mobile GIS with its relational tables were supported by the ArcGIS online platform. The ArcGIS online platform is part of the ESRI geospatial cloud system, which enables mobile and remote connection to people, groups, locations, and data using interactive maps (ESRI, 2020a). The system is embedded with smart, data-driven and analytical tools that support the delivery of real-time and near real-time location intelligence. The basic required resources included human expertise, geographically referenced data, and organization including operational protocols, hardware and software. COVID-19 data capture and management were under the surveillance task per the governance structure. The development and deployment of the geodatabase for COVID-19 management involved GIS and application, development experts. The GIS and application development experts developed the backend and relational databases. The team customized the interface of the application and set up the connectivity. Twenty Field Epidemiologists were trained on the use of the application. The Field Epidemiologists served as field coordinators and supervisors during the 
contact tracing and enhanced contact tracing. These coordinators play a supervisory role and liaise between the application development team, Senior Field Epidemiologist and the fieldworkers. The direct users of the application (fieldworkers) included selected Disease Control Officers, Public Health Officers, Field Technical Officers, Community Health Nurses, Laboratory Scientists and Lab Technicians. In all, over 200 field workers were deployed. The fieldworkers collected samples and location information from cases and contacts, to populate the database and map the absolute location of the patient. The Application Development Team (GIS Team) provided training for the coordinators and the coordinators trained the fieldworkers. The fieldworkers also report feedbacks through the coordinators. The GIS Team also trained the Laboratory Staff who test the samples and record the results into the application. The test results were visualized on Regional Directors Dashboard and Decision Makers Dashboard. The Decision Makers Dashboard is solely a decision-making platform headed by the National Coordinator of the COVID-19 Programme appointed by the president. The spatially referenced data used included google map and google image that provided the spatial reference including building footprints to help capture the absolute location of the patient. Similarly, the design and deployment followed COVID-19 protocols developed by Ghana Health Services (GHS) and the Ghana COVID-19 Monitoring Team. The protocol involved the use of case-based investigation form, protection of an individual's identity and protection of privacy and workers safety. The hardware required included laptops, cloud storage space, connectivity and workspace, which were provided by the University of Ghana, and tablets provided by the Malaria Control Program. Finally, the software used included ArcGIS Pro Desktop, ArcGIS Online, and Collector for ArcGIS and Survey 123 for ArcGIS, provided by the Remote Sensing and GIS Applications Laboratory, Department of Geography and Resource Development, University of Ghana, Legon. These hardware and software provided the complete mobile GIS system with intelligence that supported Ghana's COVID19 monitoring. The resultant ArcGIS geodatabase is a collection of geographic datasets as well as attribute information of various types held in a common file system (ESRI, 2020b; ESRI, 2020a). The geodatabase can be accessed using ArcGIS online, ArcMap and ArcCatalog as well as ArcGIS Pro. 


\section{Development of the backend relational geodatabase}

The development of the backend geodatabase (the geodatabase and related tables that form the basis of this application) was informed by the need to identify individual cases and their contacts through contact tracing and enhanced contact tracing. The backend development was also to enhance automation, visualization and analytics of test results. It is against this background that the principle of a relational database was used in customizing and standardizing the backend geodatabase, which made data accessibility and management relatively easier. The design utilized two database principles: relate and relationship classes. The related principle as created in the layer file provided a system in which when a case or household is selected in a layer, its related cases or individuals (contacts) are easily identified in the layer or related records in a non-spatial table (Boden, 2018). Practically, the principle means that when a case is identified, using the ID field, the contacts are captured as related tables (case and related contact). The system also used a relationship class where COVID-19 cases were captured as objects in the geodatabase. This relationship class design enables smart behaviour in visualizing test results on the output side, which is the decision-makers and regional directors' dashboard, in Ghana's context. Both the relate and relationship classes rely on the principle of cardinality. Cardinality in GIS describes how records in a table or two different tables are related to one another. Cardinality builds one-to-one, one-to-many, manyto-one, or many-to-many relationships in the database (Boden, 2018). The database contained COVID-19 cases locations and a non-spatial table (attribute) that stores the attribute data about the COVID-19 cases based on a case-based investigation form from GHS. A relate was created between the layer and the non-spatial table, which has a many-to-one (contacts to the patient) cardinality because every case has multiple persons serving as contacts. Relate is based on the ID field (EPI ID). The system did not focus on many-to-many (case to contact and contact to contact) because not all contacts become cases. Again, most people do not remember others they meet daily unless something remarkable happens that day. Relationship classes gave the ability to visualize data easily. With relationship class, design rules were set up to link field samples to testing labs, dashboards and analytics. When field workers go to the field and they take a sample for testing, the samples are tagged with QR codes and recorded into the online geodatabase. These samples were taken from cases, contacts of cases and individuals. The testing laboratories assess the database online after receiving the samples by scanning the $\mathrm{QR}$ code on the sample bottle to identify and authenticate the sample before testing is done. When test result fields in the database are populated with values, the system automatically colour- 
codes those fields, changes the symbology of the map and update the dashboards. The dashboards are the outputs for decision-makers by providing up to date data on the pandemic with better data visualization. The colour-coding was classified as; red (positive), blue (negative), green (sample yet to be tested) and black as inconclusive (which means the sample needs to be retaken). The embedded analytics then recalculates and updates the number of positives from regions and districts and displays the results numerically. Similarly, the sample count is also updated when a new record is taken from the field to monitor the activities of field workers.

For the Covid-19 enhanced contact tracing, field teams were required to sample residents who can be located within a $2 \mathrm{~km}$ radius of an existing case. The absolute location of the house is recorded using the collector for ArcGIS, which works and submits data directly into the geodatabase. Since a house may host more than one person, a related database table was created to contain all other information from the members of that particular household. To avoid each household individual having a spatial representation (point), a relationship was created between a table that represented household individuals and the spatial data that represented the household itself. A foreign key was used to initiate many-to-one relations to make it possible for the spatial and attribute information to communicate effectively in such a way that a click on the spatial data points to all other members (individuals) belonging to the spatial data (Household) from the relation table.

\section{The Use of the system for an enhanced contact tracing on the field}

The application was deployed on android tablets, which were carried by the fieldworkers to the field to map cases and their contacts. Besides, using the ArcGIS collector application, fieldworkers were able to see the initial 60 cases shown as red points with $2 \mathrm{~km}$ buffer rings around them. With the current location on the ground showing on the application interface, fieldworkers have a fair idea of where they were standing on the map and were able to move within the target zone. Due to internet instability, fieldworks were able to download and work offline and once data collection is done, all information was synced to the database.

\section{Evaluation of the database output}

The design and deployment of relational geodatabase on mobile geospatial technology, as used in Ghana's COVID-19 management, is seen as a system designed to capture, store, manipulate, analyze, manage, and present all types of geographical data. Geographic data consist of both 
spatial and attribute data. The completed system had two sides: the data capture (which includes the sample taking activities in the field) and the laboratory testing activities on one side, and the output side (which consists of two dashboards i.e., one for the regional and district directors of health and the other for the National COVID-19 Management Center).

At the onset, Ghana identified the need for contact tracing and surveillance for tracking the virus in real-time or near real-time. The contact tracing was later upgraded to enhanced contact tracing. Enhanced contact tracing was defined by the COVID-19 management team and was employed in this study as part of the Ghana COVID-19 management protocol as 'an advanced form of contact tracing, which deals with the testing of samples from residents within a certain specified area of an already confirmed case, to get ahead of the spread of the virus and also aid in containing the spread of the virus.' While contact tracing focuses on individuals who have had contacts with COVID-19 patients, enhanced contact tracing focuses on individuals who live within a defined physical contact space of $2 \mathrm{~km}$ from a known COVID-19 patient location. The contact tracing and enhanced contact tracing activities, as implemented in Ghana called for the usage of GIS-based technology to map all cases, their contacts and their residential location as well as households within some distance of identified cases. This was essential to allow an immediate visualization of the extent and magnitude of the public health problem and plan national scale intervention strategies (Hay, et. al. 2013; Fortunati, 2016).

The advantage of using the relational geodatabase is that it is publishable and could be used on other ArcGIS related platform for data collection, which in this case was the Collector for ArcGIS and survey 123 for ArcGIS. The geodatabase, which was created within ArcGIS Pro was then published onto ArcGIS online for further configuration to suit the need of the project. The structure of the relational database that allowed contact tracing is shown in Figure 2. The use of multiple ID fields allowed individuals identified in the database as Head of Household $(\mathrm{HH})$, linked to their respective contacts. From Figure 3, Mr John Johnson is captured as HH and he will still appear in the related records, he may not necessarily be a case. In contact tracing, he is a contact to a case. He has his contacts, which were captured as related records. When contacts become positive, they also generate new contacts, yet they keep their link with their primary contact. The use of multiple ID fields and linkage of HH to contacts and case contacts to related contacts becomes relevant to case decision-makers and field technicians for testing of contacts, monitoring of contacts and their related contacts, for follow-ups and early detection and isolation. 


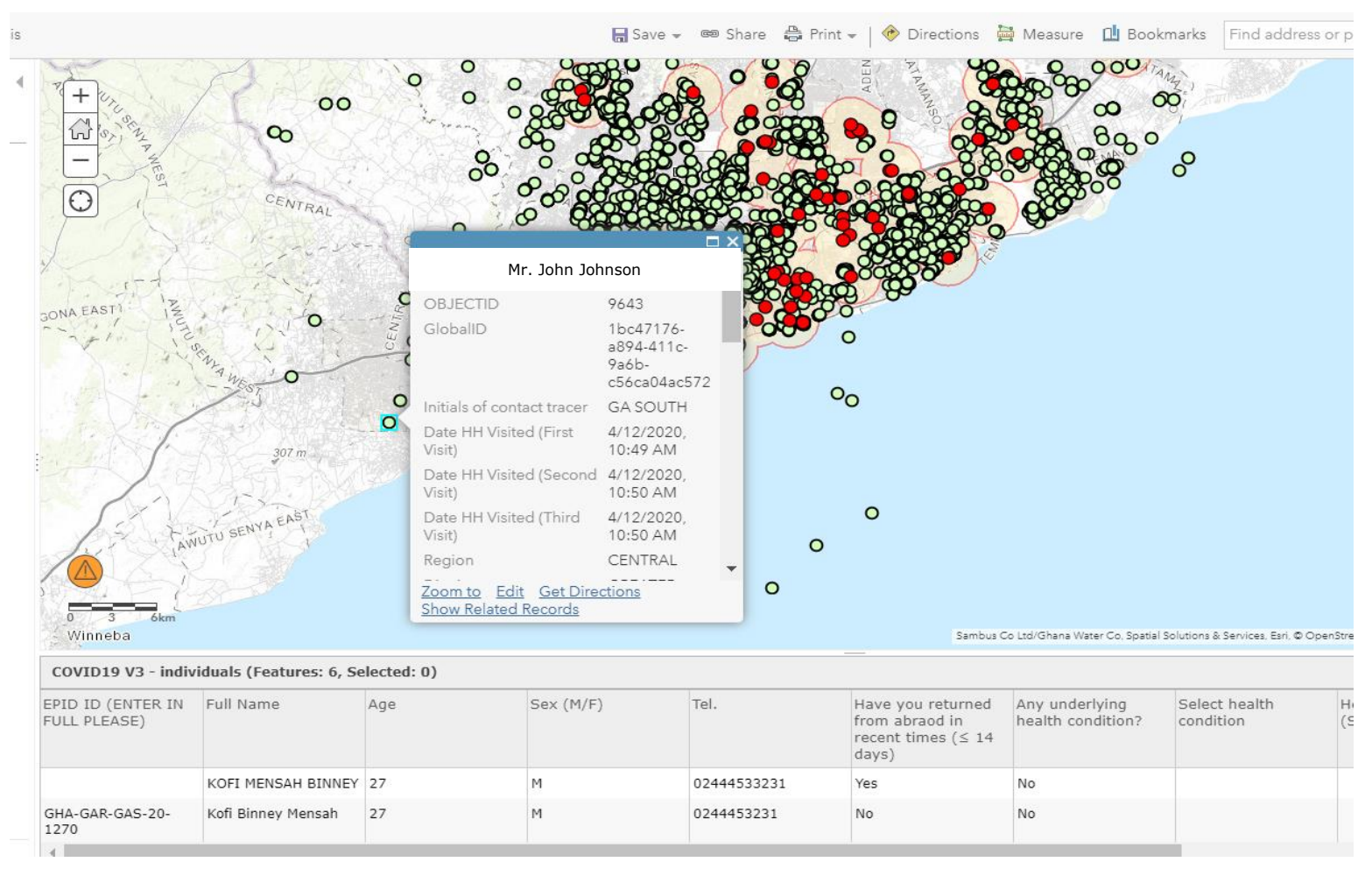

Figure 3: Overview of the Collector for ArcGIS and the backend related database Source: Author 2020

Since the application was used for two main purposes, namely: contact tracing and enhanced contact tracing, it is important to clarify that $\mathrm{HH}$ may be a case or contact for a case for contact tracing purposes. However, HH may or may not be a case nor a contact of a case if the application is being used for enhanced contact tracing. The use of contact tracing and enhanced contact tracing were premised on the objective of being ahead of the spread of the virus. Individual's location and attributes were captured irrespective of what the possible outcome may be (conversion to the case or not). In addition, when individuals are sampled and mapped as $\mathrm{HH}$, they are identified in the database as a related table and therefore become individual contacts themselves since a set is a subset of itself. Mapping samples such as $\mathrm{HH}$ means that when counting samples taken with the app, $\mathrm{HH}$ is not counted to avoid double counting. This approach was useful in the identification of cases and contacts to cases within the database. The approach also allowed add-ons when new contacts are identified. Within the database, a search is done based on Case-based ID and connected individual ID and these two help in identifying all contacts to $\mathrm{HH}$. Figure 4 depicts contact tracing using a contact tree as implemented in the relational database model. 


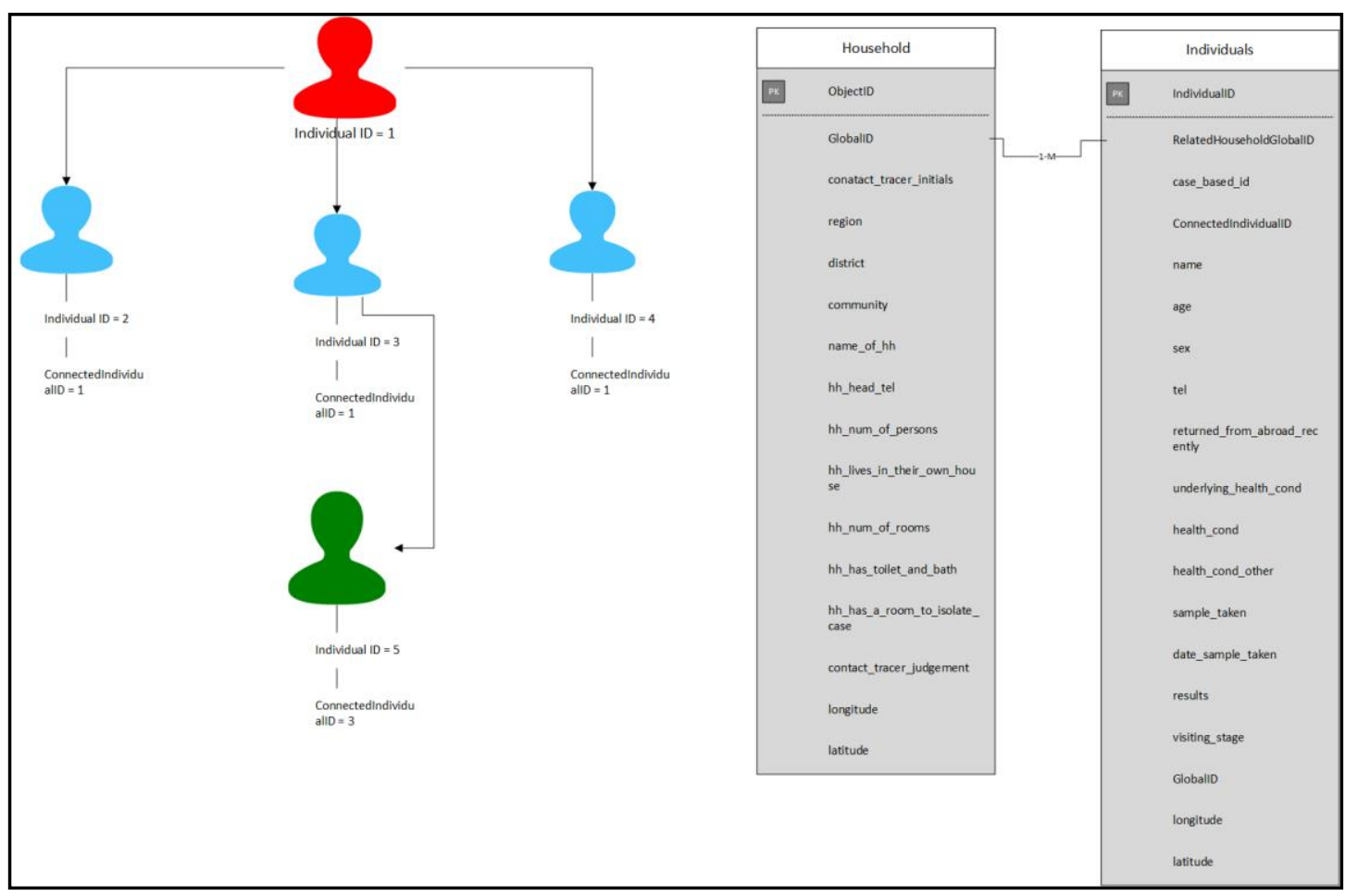

Figure 4: Contact Tracing and Community Case Tracking Relational Tables Source: Author 2020

Before the launching of the application, fieldworkers had taken samples within some selected communities. Each metropolitan and municipality developed their Epi ID, which uses 15 alphanumeric characters beginning with three (3) letter country code (GHA), three (3) letter region code (GAR), three-letter Municipality of Metropolitan code (AMA) and six (6) digit case unique number.

A review of the relational geodatabase application shows that by $4^{\text {th }}$ April 2020, barely four (4) days after deployment, 828 samples had been collected using the application. The test results indicated that 34 new cases were found. Out of the 34 cases, $61 \%$ occurred outside the $2 \mathrm{~km}$ buffer, which shows the spread of infections beyond physical neighbourhood contact, i.e. rejection of the Null hypothesis: that Covid-19 will spread only by physical neighbourhood contacts during the lockdown period. The above results suggest that cases did not necessarily spread through physical community contact only. Several reasons may have contributed to this result. It is possible, that there were more cases than the original known 60 cases within the country, which were not known as of the time of the lockdown. Besides, infected individuals might have travelled outside their communities. Figure 5 shows the 60 initial cases in red dots with its $2 \mathrm{~km}$ buffer, defining the physical neighbourhood contact space. Cases during the first 
few days of the lockdown are green and occurred either within or outside physical contact space.

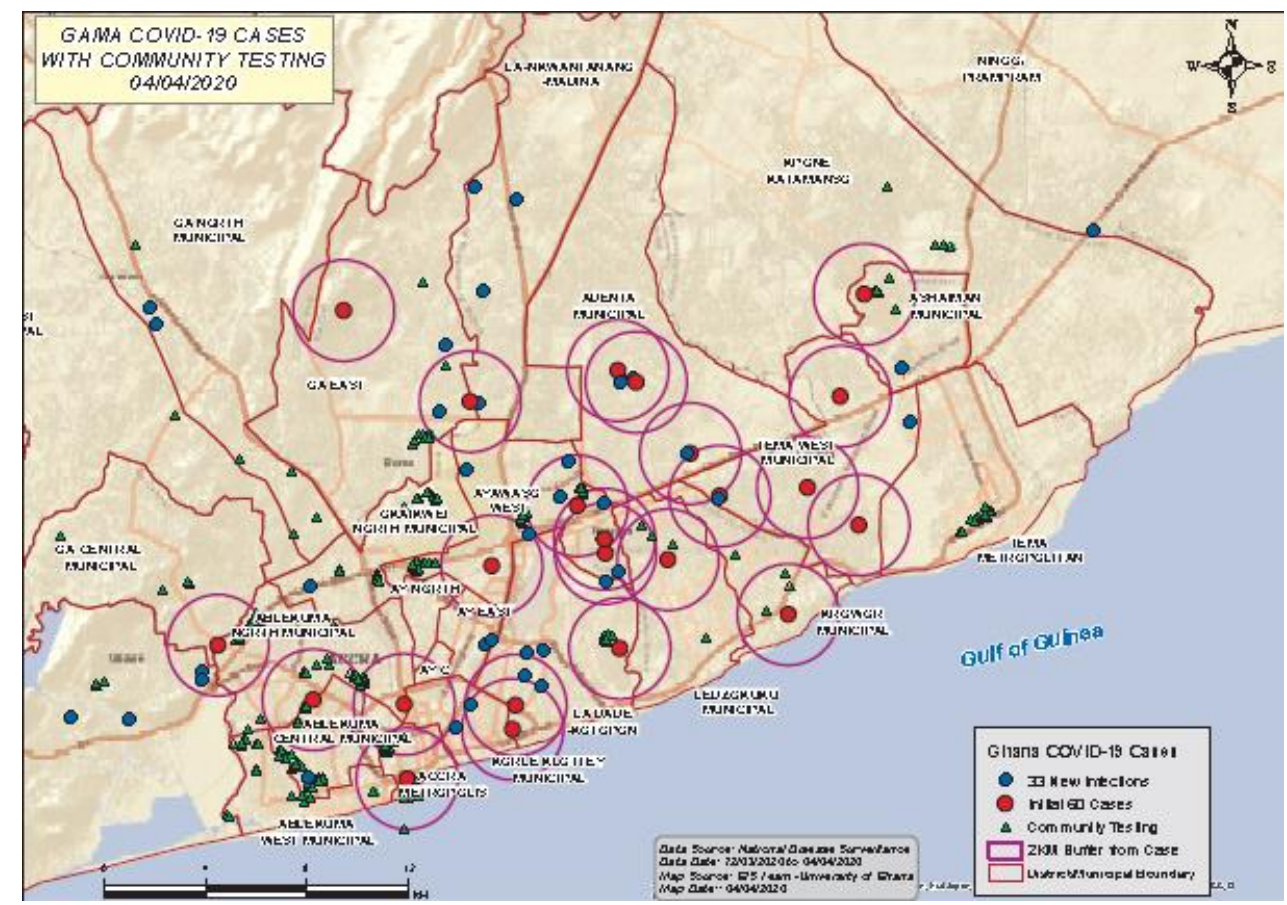

Figure 5: COVID-19 Cases in GAMA 5 days after lockdown Source: Author 2020

The contact tracing was then augmented with the enhanced contact tracing activity using both the Collector for ArcGIS and Survey 123 for ArcGIS applications. By April 30 ${ }^{\text {th }}$ 2020, a total number of 8,748 individuals identified and mapped as head of households $(\mathrm{HH})$ in the database were sampled as possible community contacts. These heads of households had 16,087 contacts linked to them as related individuals. It is important to note that these $\mathrm{HH}$ and their related contacts were not necessarily cases from the start. This was Ghana's approach, which sought to move ahead of the spread of the virus, by providing important baseline estimates of the pandemic, detecting and isolating infected individuals before the virus spreads to others and targeting resource allocation (GHS, 2020; Pigott et. al., 2015). After testing the 8,748 individuals, $388(2.4 \%)$ turned out to be positive. From the initial observation that $61 \%$ of infections occurred outside physical neighbourhood contacts, attention was shifted from sampling within the $2 \mathrm{~km}$ buffer, which is then defined as physical neighbourhood contacts to locations outside the case community and around the new cases outside the community. This spatial attention shift was informed by the suspicion that interactions are probably taking place at a wider GAMA community than the neighbourhood. In total 7,501 $\mathrm{HH}$ were sampled and they had 17,071 individuals as contacts, among which 741 (4.3\%) came out positive. This result 
means that while attention was being drawn to within community spread at the initial stage, more infections were taking place between communities. The above finding underscores the direction of transmission, outbreak tracking and risk mapping as some of the key importance of geospatial technology application in infectious disease surveillance such as COVID 19 (Pigott et. al., 2015; Saran et. al.,2020).

Figure 6 is the map of Ghana showing the lockdown areas and location of COVID-19 cases as of $30^{\text {th }}$ April, 2020 when the lockdown was lifted. It was observed that even with the enhanced contact tracing at lockdown areas, cases were reported outside the areas. The purpose of the lockdown was to prevent cases from spreading to other regions and keep the cases within the infected communities in the lockdown area. The data from the enhanced contact tracing found that over $60 \%$ of new infections occurred within the lockdown areas but outside the initially infected communities. The distribution of cases before and after the lockdown in GAMA is shown in Table 1.

Table 1: COVID-19 Cases before and at the end of Lockdown (Source: Author 2020)

\begin{tabular}{llll}
\hline Metropolitan /Municipality & $\begin{array}{l}\text { Cases } \\
\text { Lockdown }\end{array}$ & $\begin{array}{l}\text { Before } \\
\text { of lockdown }\end{array}$ & $\begin{array}{l}\text { Casea the end } \\
\text { Square KM }\end{array}$ \\
\hline Adenta Municipal & 5 & 17 & 79 \\
Ledzokuku Municipal & 1 & 16 & 30 \\
La Dade-Kotopon & 5 & 29 & 37 \\
La-Nkwantanang-Madina & 3 & 26 & 72 \\
Ga East & 2 & 54 & 87 \\
Ayawaso West & 10 & 61 & 33 \\
Ga South Municipal & 0 & 2 & 181 \\
Ga West Municipal & 0 & 13 & 178 \\
Ga Central Municipal & 0 & 31 & 50 \\
Tema West Municipal & 4 & 15 & 46 \\
Ashaiman Municipal & 2 & 15 & 19 \\
Kpone Katamanso & 1 & 18 & 244 \\
Ablekuma Central Municipal & 1 & 191 & 14 \\
Korle Klottey Municipal & 3 & 14 & 10 \\
Ablekuma North Municipal & 1 & 71 & 30 \\
Ayawaso North Municipal & 0 & 128 & 5 \\
Ayawaso East Municipal & 3 & 113 & 6 \\
Okaikwei North Municipal & 0 & 34 & 23 \\
Ga North Municipal & 3 & 25 & 127 \\
Weija Gbawe Municipal & 3 & 38 & 166 \\
Krowor Municipal & 0 & 19 & 19 \\
Tema Metropolitan & 0 & 26 & 43 \\
Ablekuma West Municipal & 1 & 85 & 13 \\
Ayawaso Central Municipal & 0 & 112 & 2 \\
Accra Metropolis & 0 & 91 & 6 \\
\hline
\end{tabular}


At the beginning of the lockdown, Ayawaso West, a typical high-class residential neighbourhood, had the highest number of cases (10 people were positive) followed by La Dadekotopon and Adenta (5 each) and Tema West (4). Interestingly all these metropolitan areas are high-income residential areas. At the end of the lockdown, Ablekuma Central, Ayawaso Central, Ayawaso North and Ayawaso East were the leading metropolitan areas. Surprisingly all these four (4) Metro areas are low-income residential areas where people must work daily to feed themselves and their families. In addition, the new leading areas were areas where police had challenges enforcing lockdown and social distancing. Figure 6 visualizes the spatial distribution of cases in Ghana with an inset of the two-lockdown areas: GAMA and GKMA. The study found that whiles GAMA and GKMA were locked down resulting in control of the spread of the virus; infections were taking place in other areas, which were not locked down. Again, with the enhanced contact tracing, efforts were made to track the virus and be ahead of the virus by looking for local community (physical contact space) contacts, yet infections were beyond the local community contacts defined. This finding suggests that the nature of the virus and the observed large number of asymptomatic cases in the country made it impossible to track cases before the lockdown when the geospatial technology was not in use. Perhaps the limited mobility allowed during the lockdown may have played a role in why cases spread to other places without known cases before the lockdown.

On the output side of the application, we realized the value of the design and deployment of the relational geodatabase on mobile geospatial applications for contact tracing activities in terms of the testing laboratories communication with fieldworkers and decision-makers in realtime. The laboratories accessed the database and used the application for planning staff schedule before samples were received. The samples were scanned and recorded using the application. Upon completion of the test, the database is updated with test results, which automatically updates and symbolizes the visualization dashboard with the test results. The dashboard is an interactive map that locates and tallies confirmed cases, recoveries and deaths. The virus infection progress details were shown by graphs. Viewers can see the day and time of the most recent data update and data sources (Boulos and Geraghty, 2020). This approach was very useful in improving contact tracing, community targeting, communication and decision-making. 


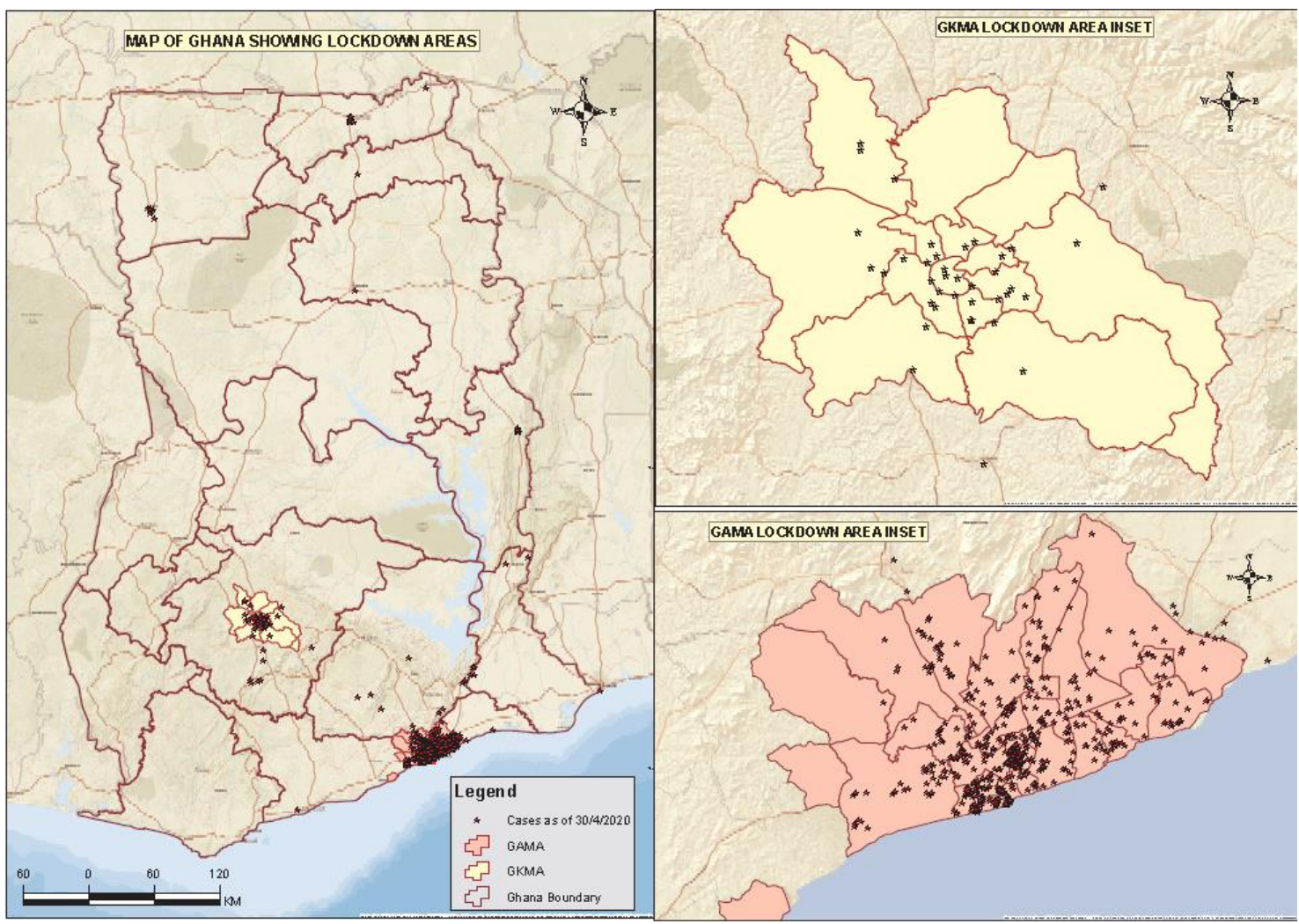

Figure 6: Map of Ghana showing the lockdown areas and location of cases as of $30^{\text {th }}$ April, 2020 Source: Author 2020

The applications allowed rapid response to communities with new outbreaks. The applications also helped in understanding and monitoring the geographic spread and modification of approach to responding to COVID-19 pandemic more appropriately than would have been traditionally (Boulos and Geraghty, 2020; ESRI, 2020c). The application provided real-time reporting and communication between field workers, testing laboratories, regional health directorates and national-level decision-makers.

\section{Conclusion and Recommendation}

Ghana's approach in managing COVID-19 was motivated by the desire to be ahead of the spread of the virus through early detection, effective contact tracing and case isolation to stop the spread of the virus to other locations in the country. This approach formed the basis of the lockdown, the contact and enhanced contact tracing activities. However, these approaches were initiated without a clearer understanding of the country's COVID-19 status in terms of geographically referenced data on cases, case characteristics and spread. The development of the relational geodatabase, which runs on mobile geospatial applications, was to help implement the planned strategy. The mobile geospatial applications, which were developed 
included the collector for ArcGIS and Survey 123 for ArcGIS to enable field workers to collect samples and to map the location of the cases and samples taken for real-time reporting. These applications also allowed the integration of bar code systems for easy access and entry of data. The relational geodatabase on geospatial applications also allowed the development of a Laboratory portal for the testing labs to access field records, enter test results, and communicate results to regional directors for case management. Two dashboards, the decision-makers dashboard and regional directors' dashboard were created. Regional directors were able to show the location of cases within their respective regions on daily bases for quick action. The Decision Makers' dashboard on the other hand allowed decision-makers, e.g., to comprehensively have a national view of cases. The dashboard for Decision Makers shows the district and regional breakdown of cases, the spatial pattern of cases and the temporal pattern of spread.

In addition, the design of the relational database for contact tracing and surveillance supported the early understanding that cases were not spreading necessarily from the location of the known case outward. This observation meant that the alternative proposition might better explain the COVD-19 situation. Many reasons could have possibly contributed to this observation; that: 1) some unknown cases existed in the country before the lockdown; 2) some individuals were positive and were moving and spreading infections to other locations in the country where there were no known cases; 3 ) It is also possible that several positive individuals entered the country through unapproved routes. This third argument is being made against the background that the lockdown occurred in areas with known cases and that importation of cases ceased with the closure of the country's international borders. This conclusion is supported by the fact that new cases were reported in regions not known to have reported COVID-19 cases such as; the Upper East Region, the Upper West region, Eastern region, Savannah region, Oti and Volta regions and the Central region. The COVID-19 monitoring team used this mobile GIS application to map 10 reported cases in the northern region of Ghana and they were identified as imported cases that used unapproved routes in entering the country. Another possible argument was that the time lag between the announcement and enforcement of the lockdown led to 'panic' travel outside the lockdown area. This panic travel could have spread the virus to places, hitherto, were not considered COVID-19 endemic.

The study observed that COVID-19 cases started from high-income residential areas and spread to low-income neighbourhoods. Interestingly, cases from low-income neighbourhoods 
could not be linked to specific case (s) from high-income neighbourhood, as people could not remember their contacts and sources of infection. This made it difficult to explain the infection sources, types and patterns for specific action to either reduce or stop the spread. It was therefore not surprising that experts explained the reasons for ending the lockdown as economically motivated. In low-income neighbourhoods, social distancing was not observed; masks were in limited use and above all people worked daily for survival even under lockdown conditions.

Based on these findings from a relational geodatabase, this study makes the following observations that Ghana's approach to managing COVID-19 would have slowed the spread of the virus at the onset if the lockdown were instituted nationwide. There should have been a total lockdown with no movement allowed. Stringent measures were needed to prevent the importation into the country through unapproved routes. However, the counter-arguments raised against the total lockdown of the entire country with a complete restriction on movements were that the economic and social cost would have been unbearable given the economic situation and the spatial organization of the country.

The study has demonstrated the usefulness of relational geodatabase for contact tracing, realtime Spatio-temporal monitoring of COVID-19 and communication between field workers, testing labs and regional and national level decision-makers. Future works may focus on 1) the design and deployment of relational geodatabase that assesses many to many relations as this work focused entirely on many-to-one relations. 2) Design and deployment of an integration case and logistics management information in the relational geodatabase for a comprehensive one-stop-shop geospatial system for disease control. 


\section{References}

AS. (2020). Coronavirus: what have been the worst pandemics and epidemics in history? Available at: ttps://en.as.com/en/2020/04/18/other_sports/1587167182_422066.html. Accessed: July 9, 2020.

Boden, S. (2018). Relates and Relationship Classes Explained. Available at: https://www.esri.com/about/newsroom/wp-content/uploads/2018/11/relatesrelationship-classes-explained.pdf. Accessed: July 17, 2020.

Boulos, M.N.K., \& Geraghty, E.M. (2020). Geographical tracking and mapping of coronavirus disease COVID-19/severe acute respiratory syndrome coronavirus 2 (SARS-CoV-2) epidemic and associated events around the world: how 21st century GIS technologies are supporting the global fight against outbreaks and epidemics. Int $J$ Health Geography. 2020 Mar 11;19(1):8. doi: 10.1186/s12942-020-00202-8.

Chatham House. (2020). The Hurdles to Developing a COVID-19 Vaccine: Why International Cooperation is Needed. Available at:https://www.chathamhouse.org/expert/comment/hurdles-developing-covid-19vaccine-why-international-cooperationneeded?gclid=Cj0KCQjwo6D4BRDgARIsAA6uN1tCU6fRdeamg49mma9ai2RZ_zQwbon_gYyzeSpiK16rQ_LvbtMbEaAlUrEALw_wcB. Accessed: July 10, 2020.

Chaturvedi, A. (2020). Coronavirus: Geospatial industry pitches in to help combat pandemic. Available at: https://www.geospatialworld.net/blogs/initiatives-by-geospatialcompanies-to-curb-coronavirus/. Accessed: July 03, 2020.

Chung, D., \& Hoon, S. S. (2020). Korea's response to COVID-19: Early lessons in tackling the pandemic. Available at: https://blogs.worldbank.org/eastasiapacific/koreasresponse-covid-19-early-lessons-tackling-pandemic. Accessed: July 04, 2020.

ESRI. (2020a). ArcGIS Pro. Available at: https://pro.arcgis.com/en/proapp/help/data/geodatabases/overview/what-is-a-geodatabase.htm\#: :text=At\%20its\%20most\%20basic\%20level,\%2C\%20PostgreSQL\%2C\%20or \%20IBM\%20DB2.\&text=But\%20a\%20geodatabase \%20is\%20more\%20than $\% 20 \mathrm{a} \% 2$ 0collection\%20of\%20datasets. Accessed: July 17, 2020.

ESRI. (2020b). COVID-19. Maps, solutions, and resources for response and reopening. Available at: https://www.esri.com/en-us/covid-19/overview. Accessed: July 17, 2020.

ESRI. (2020c). COVID-19 CDC Supports State Dashboard to Better Monitor cases and capacity. https://www.esri.com/about/newsroom/blog/covid-19-cdc-supports-statedashboards-to-better-monitor-cases-and-capacity/ Accessed: July 19, 2020.

Fortunati, R. (2016). Mapping Ebola to prepare for future outbreaks. IHME Measuringwhat matters . Retrieved from http://www.health data.org/acting-data/mapping-ebolaprepare-future-outbreaks. Accessed: July 19, 2020.

GHS. (2020). Situation Update, Confirmed COVID-19 Cases In Ghana As At 27 March 2020, 19:00 Hr. Available at: https://ghanahealthservice.org/covid19/archive.php. Accessed: July $15,2020$. 
Hay, S.I., Battle, K.E., Pigott, D.M., Smith, D.L., Moyes, C.L., Bhatt, S., Brownstein, J.S., Collier, N., Myers, M.F., George, D.B., \& Gething, P.W. (2013). Global mapping of infectious disease. Philosophical Transactions of the Royal Society B: Biological Sciences, 368(1614), p.20120250.

Herold, S., \& Sawada, M. C. (2012). A review of geospatial information technology for natural disaster management in developing countries. International Journal of Applied Geospatial Research (IJAGR), 3(2), 24-62.

ILO. (2020). COVID-19 and the world of work - Country policy responses. Available at: https://www.ilo.org/global/topics/coronavirus/country-responses/lang--en/index.htm. Accessed: July 9, 2020.

Malgorzata, D., \& Pomfret, D. K. (2020). Geospatial information management in the time of COVID-19. Available at https://www.geospatialworld.net/blogs/geospatialinformation-management-in-the-time-of-covid-19/. Accessed: July 03, 2020.

Nkansah, M. A. (2020). [Case Study] Ghana's multifarious response to COVID-19: Through a citizen's lens. Available at: https://www.ingsa.org/covidtag/covid-19commentary/asantewah-nkansah-ghana/. Accessed: July 15, 2020.

Pigott, D.M., Howes, R.E., Wiebe, A., Battle, K.E., Golding, N., Gething, P.W., Dowell, S.F., Farag, T.H., Garcia, A.J., Kimball, A.M., \& Krause, L.K. (2015). Prioritising infectious disease mapping. PLoS Neglected Tropical Diseases, 9(6), p.e0003756

Ritchie, H., Ortiz-Ospina, E., Beltekian, D., Mathieu, E., Hasell, J., Macdonald, B., Giattino, C., Roser, M., Yunits, van Woerden, E., Gavrilov, D., Bergel, M., Ahmad, S., \& Crawford, J. (2020). Policy Responses to the Coronavirus Pandemic. Available at: https://ourworldindata.org/policy-responses-covid. Accessed: July 9, 2020.

Saran, S., Singh, P., Kumar, V., \& Chauhan, P. (2020). Review of Geospatial Technology for Infectious Disease Surveillance: Use Case on COVID-19. Journal of the Indian Society of Remote Sensing, pp.1-18.

Torhonen, M. K., Antos, E. S, Dongkyu, K., \& Alvaro B. F. (2020). The role of geospatial information in confronting COVID-19 - Learning from Korea. Available at:https://blogs.worldbank.org/eastasiapacific/role-geospatial-informationconfronting-covid-19-learning-korea. Accesses: July 04, 2020.

US Census Bureau, (2015). Basics on Geodatabases. Available at: http://ggim.un.org/meetings/2015-ISGI-Jordan/documents/Session\%203-

Geodatabases.pdf. Accessed: July 15, 2020.

WHO. (2020). Coronavirus Disease (COVID-19) Dashboard. Available at https://covid19.who.int/?gclid=CjwKCAjwi_b3BRAGEiwAemPNU389V27iQ9nbQ1 bGjRge8qW1sBBhDYGrZO1UbKltTSHoMXdH0Ke35RoCWngQAvD_BwE July 02, 2020. Accessed: July 2020.

Xu, E. N., \& Chandler, C. (2020). When red is unlucky: What we can learn from China'scolourcoded apps for tracking the coronavirus outbreak. Available at: https://fortune.com/2020/04/20/china-coronavirus-tracking-apps-color-codes-covid19-alibaba-tencent-baidu/. Accessed: July 04, 2020. 